\section{Management of childhood eczema: reflections from secondary care}

From my experience in secondary care, managing parental expectations and helping families integrate treatment plans into their daily routine are some of the key challenges in managing childhood eczema. Clinicians have to rely on their professional judgement when counselling families on the safe use of topical corticosteroids as guidelines do not provide clear recommendations. Many primary care physicians in the UK may find themselves not in a position to initiate topical calcineurin inhibitor as a steroid sparing therapy due to NICE guidance. It is important to explore the family's treatment preference and their daily skin care routine for the affected child. Recurrent themes emerging from my discussion with families on opportunities to simplify and optimise treatment regime include:

- Navigating the treatment ladder: families may be advised to gradually cut down the frequency of a topical corticosteroid therapy (twice daily $>$ once daily $>$ alternate days > stopl as eczema improves, instead of stepping down the potency to reduce the number of prescription items they have to remember to use.

- Treatment duration: families should be advised to treat an area of eczema until its full resolution as premature stopping of therapy contributes to eczema flare up.

- Choice of emollients: families may be shown or given samples (if available) of different consistency of emollients to try before selecting a preferred emollient for regular use to minimise wastage.

- Education: families may be shown how to recognise lichenified areas of eczema, which may require prolonged topical corticosteroid therapy lor topical calcineurin inhibitor therapyl until the lichenified areas become smooth again. Topical treatments should be applied in one smooth direction. There should be at least 30-minute interval between each topical therapy lor wait until the skin dries fullyl to ensure adequate absorption.

I further agree with Powell and colleagues that a written action plan has the potential to overcome some of the challenges of managing eczema in primary care. ${ }^{1}$

Simon Tso

Dermatology ST6, University Hospitals Coventry and Warwickshire NHS Trust, Coventry.

Email: simontsoladoctors.org.uk

\section{REFERENCE}

1. Powell K, Le Roux E, Banks JP, Ridd MJ.

Developing a written action plan for children with eczema: a qualitative study. Br J Gen Pract 2018; DOI: https://doi.org/10.3399/bjgp17X693617.

DOI: https://doi.org/10.3399/bjgp18X696341 